\title{
Spheroid-Based Approach to Assess Tissue Relevance of Analysis of Dispersed-Settled Tissue Cells by Cytometry of Reaction Rate Constant
}

\author{
Vasilij Koshkin, Mariana Bleker de Oliveira, Chun Peng, Laurie E. Ailles, Geoffrey Liu, Allan Covens, \\ and Sergey N. Krylov*
}

Supporting information placeholder

ABSTRACT: Cytometry of Reaction Rate Constant (CRRC) uses time-lapse fluorescence microscopy to measure a rate constant of a catalytic reaction in individual cells and, thus, facilitate accurate size determination for cell subpopulations with distinct efficiencies of this reaction. Practical CRRC requires that a tissue sample be disintegrated into a suspension of dispersed cells and these cells settle on the support surface before being analyzed by CRRC. We call such cells "dispersed-settled" to distinguish them from cells

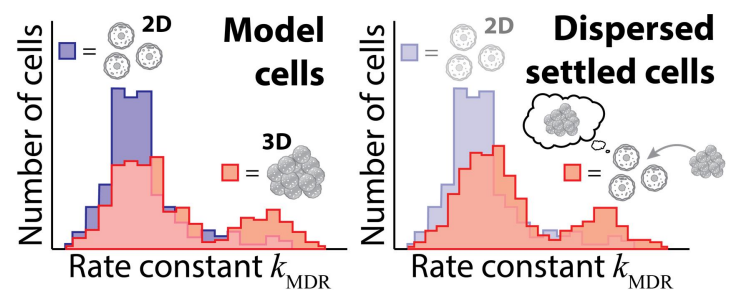
cultured as a monolayer. Studies of the dispersed-settled cells can be tissue-relevant only if the cells maintain their 3D tissue state during the multi-hour CRRC procedure. Here we propose an approach for assessing tissue relevance of the CRRC-based analysis of the dispersedsettled cells. Our approach utilizes cultured multicellular spheroids as a 3D cell model and cultured cell monolayers as a 2D cell model. The CRRC results of the dispersed-settled cells derived from spheroids are compared to those of spheroids and monolayers in order to find if the dispersed-settled cells are representative of the spheroids. To demonstrate its practical use, we applied this approach to a cellular reaction of multi-drug-resistance (MRD) transport which was followed by extrusion of a fluorescent substrate from the cells. The approach proved to be reliable and revealed long-term maintenance of MDR transport in the dispersed-settled cells obtained from cultured ovarian cancer spheroids. Accordingly, CRRC can be used to determine accurately the size of a cell subpopulation with an elevated level of MDR transport in tumor samples, which makes CRRC a suitable method for the development of MDR-based predictors of chemoresistance. The proposed spheroid-based approach for validation of CRRC is applicable to other types of cellular reactions, and, thus, will be an indispensable tool for transforming CRRC from an experimental technique into practical analytical tool.

It is well known that cell populations within the same tissue are inherently heterogeneous. ${ }^{1}$ Differences between the cells may be so large that distinct subpopulations become identifiable within a heterogeneous cell population. ${ }^{2}$ For instance, populations of cancer cells typically consist of at least two functionally-distinct subpopulations. Bulk tumor cells constitute the majority of cells in a tumour, and they are often sensitive to chemotherapy. ${ }^{3}$ Tumor-initiating cells, in contrast, establish a relatively small subpopulation that is often resistant to cytotoxic drugs and believed to be responsible for clinical chemoresistance. ${ }^{4}$

It is obvious that cell-population heterogeneity is caused by differences in molecular reactions between the cells, and thus, must be studied by advanced methods of analytical chemistry. When cell-population heterogeneity is associated with a specific cellular reaction, this reaction can serve as a foundation for characterizing the associated cell-population heterogeneity. ${ }^{5}$ Further, if a kinetic mechanism of this reaction is known and proven to be correct in the cellular context, then a reaction rate constant can serve as a parameter that most accurately characterizes the efficiency of the reaction in a cell. Cytometry of Reaction Rate Constant (CRRC) is a general approach in which measuring a reaction rate constant in individual cells is utilized to characterize cell population heterogeneity. ${ }^{6}$

CRRC is still in its infancy. The general concept of CRRC is illustrated in Figure $1 .^{6}$ The cells are loaded with a reaction substrate, which is fluorogenic for chemical reactions and fluorescent for transport processes. Substrate conversion into a product (for transport processes, the product is simply a displaced substrate) is followed by time-lapse fluorescence microscopy at the single-cell level. The dependence of intracellular fluorescence intensity on time is used to build kinetic traces characterizing the reaction in individual cells. A reaction rate constant is determined for every cell from these kinetic traces by using a known kinetic mechanism of the reaction. A kinetic histogram "number of cells $v s$ rate constant" is built and used to determine parameters of cellpopulation heterogeneity, such as the sizes of cell subpopulations with distinct efficiencies of the cellular reaction studied.
The measurement and analysis procedures involved in CRRC are relatively elaborate and raise a question of benefits that CRRC can provide in exchange for the cost of complexity. The nonarbitrary nature of the rate constant as a measure of cellular reaction efficiency suggests a priori that CRRC should be accurate and robust, and recent experiments proved this hypothesis. It was demonstrated that CRRC can provide accurate and robust characterization of heterogeneous cell populations, while the non-kinetic characterization (utilizing classical nonkinetic cytometry histograms "number of cells $v s$ fluorescence intensity") is both inaccurate and non-robust. ${ }^{6}$ The accuracy and robustness of CRRC suggest it as a potential tool for development of reliable predictive biomarkers of cancer outcomes based, for example, on the correlation between a clinical endpoint, such as time to tumor progression, and the size of the drug-resistant subpopulation in a tumour sample.

Although many studies have identified numerous genomic, proteomic, and metabolomics alterations in cancer cells, only a few of such alterations led to FDA-approved predictive biomarkers of cancer outcomes. ${ }^{8-10}$ The lack of robust and rugged analytical techniques is among the major reasons for the alarming disagreement between the numbers of putative and approved biomarkers. ${ }^{11}$

The application of CRRC to cancer biomarker development requires the analysis of samples of tumor tissues and casts doubt on the technical implementation of such analyses. To be accurate and robust, CRRC requires (i) uniform delivery of an exogenous substrate to all cells, (ii) negligible optical interference between the cells, and (iii) stability of cells' positions during relativelylong time-lapse imaging. The first two requirements are obviously difficult to satisfy for tightly-organized multilayered cellular structures, such as samples of solid tissues. In contrast, dispersed tissue cells which are allowed to settle on a surface (which we will term, dispersed-settled cells) satisfy all three requirements and suit CRRC. Evidently, the results of studying a disintegrated tissue sample will only be tissue-relevant if the dispersed-settled cells maintain their $3 \mathrm{D}$ state (with regards to the reaction of interest) during the entire CRRC procedure. 


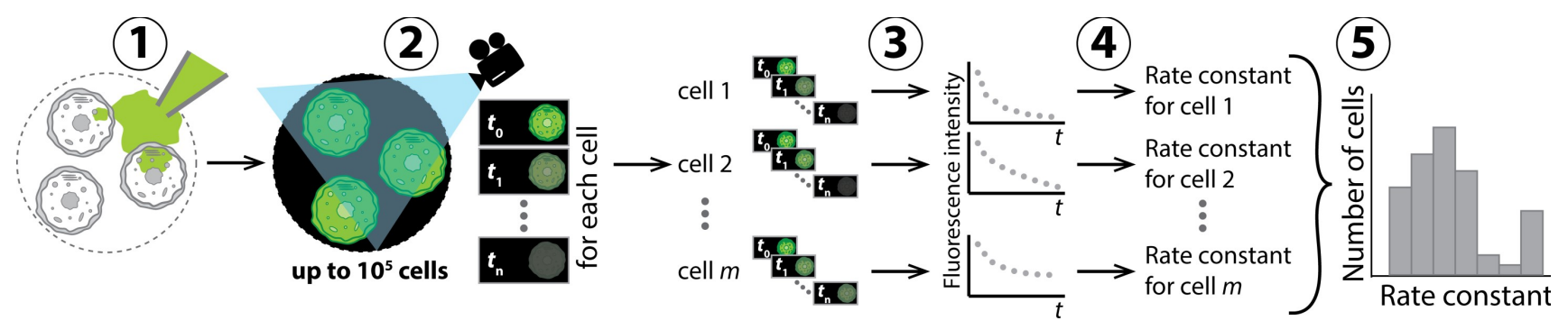

Figure 1. Schematic depiction of CRRC. A fluorescent (or fluorogenic) substrate of the reaction of interest is added to the cells (1). Kinetics of fluorescence intensity is measured microscopically - sequential images of individual cells are taken over a period of time (2). Values of the reaction rate constant are determined for each cell ( 3 and 4$)$. These values are used for a "rate constant value $v s$. number of cells" histogram (5). The heterogeneity of the population can be characterized accurately using this robust histogram. Adapted from Koshkin et al. 2019 [6].

Using CRRC for the analysis of disintegrated tissue samples requires that cell's long-term maintenance of the $3 \mathrm{D}$ state be demonstrated or at least justifiably assumed. It should be noted that this requirement is not unique to CRRC, as analyses of disintegrated tissue samples by flow cytometry also assume such long-term maintenance. ${ }^{12,13}$ Tissue disintegration into a single-cell suspension followed by cells' attachment to the surface drastically change the cells' environment. In response to this change the cells' phenotype transitions from the $3 \mathrm{D}$ state to the $2 \mathrm{D}$ state. $^{14}$ The long-term maintenance, thus, refers to the case when the characteristic transition time from the $3 \mathrm{D}$ state to the $2 \mathrm{D}$ state is much longer than the time required for tissue disintegration, cells' settling on the surface, and time-lapse imaging. Different cellular phenotypes have different transition times, and, accordingly, the long-term maintenance should be demonstrated for every phenotype individually. ${ }^{15,16}$

This work focuses on a phenotype that is associated with cross-membrane transport of xenobiotics (e.g. chemotherapeutic agents) which is catalyzed by membrane proteins known as ATPbinding cassette (ABC) transporters. ${ }^{17}$ This transport contributes to clinical multi-drug resistance (MDR) in cancer patients, and is viewed as a potential foundation for the development of chemoresistance predictors. ${ }^{18}$ The MDR-transport reaction has a known "intermediate-complex" mechanism, and, thus, can be used as a basis for the population-heterogeneity analysis by CRRC. ${ }^{19}$ Our group extensively studied MDR transport by CRRC. ${ }^{6,20-25}$ We confirmed that this reaction followed the Michaelis-Menten kinetics, and we concluded that a pseudo-first order rate constant $k_{\mathrm{MDR}}=V_{\max } / K_{\mathrm{M}}$ (where $V_{\max }$ is the maximum velocity, and $K_{\mathrm{M}}$ is the Michaelis constant) was the most convenient measure for building kinetic cytometry histograms "number of cells $v s$ rate constant" for MDR transport. ${ }^{23,25}$ Recent experiments demonstrated that CRRC could accurately and robustly distinguish two cell subpopulations with different values of $k_{\mathrm{MDR}}{ }^{6}$

With the above background on MDR transport in mind, we can now formulate the main question of this work: "Can CRRC analysis of MDR transport in a disintegrated tissue sample reveal tissue-relevant characteristics of cell-population heterogeneity?" Of course, this question can be extended to any other cellular reaction suitable for CRRC. To answer this question one needs to compare CRRC-measured heterogeneity of an intact tissue to that of the dispersed-settled cells. As we explained above, CRRC can hardly be reliably and routinely applied to intact tissue samples; CRRC of an intact tissue sample has not been demonstrated. However, nothing prevents us from an attempt to demonstrate such application for a sole purpose of comparing CRRC of an intact tissue sample with CRRC of dispersed-settled cells and answering our major question.

Such an attempt requires working with a well-controlled sample. Tissue samples from patients do not provide the required level of control. Therefore, we decided to work with cultured multicellular spheroids which are widely accepted as an adequate 3D model of solid tissues. ${ }^{26}$ The spheroids can be reproducibly cultured under controlled conditions. ${ }^{27}$ Further, the same type of immortalized cells often can be cultured both as multicellular spheroids and classical cell monolayers, ${ }^{28,29}$ allowing for the comparison of the dispersed-settled sells derived from the spheroids not only to the spheroids but also to the monolayers. Cultured monolayers can serve as a reference standard for the 2D state to which the dispersed-settled cells are expected to transition after spheroid disintegration. In essence, our spheroid-based approach for proving tissue relevance of the CRRC-based analysis requires comparing kinetic cytometry histograms of the dispersedsettled cells obtained by disintegrating cultured spheroids to those of the intact cultured spheroids (3D state) and cultured monolayers (2D state).

It is instructive to establish initially whether the histograms of intact spheroids and monolayers differ from each other. Finding a clear difference between them has two implied advantages. First, the ability to register this difference would indicate that CRRC is sufficiently precise for obtaining kinetic histograms. Second, the span of this difference constitutes a range within which the histogram of dispersed-settled cells can vary; the larger range facilitates better placement of this histogram between the 3D and 2D states. The histogram of the dispersed-settled cells is expected to change with time from that of the intact spheroids to that of the monolayers. If the histogram of the dispersed-settled cells is close to that of the intact spheroids during the entire CRRC procedure, then CRRC analysis of the dispersed-settled cells is tissuerelevant. If the histograms of the intact spheroids and monolayers are found to be similar, and CCRC is proven by another means to be precise, a conclusion can be made that the reaction of interest has similar efficiency in 3D and 2D states. In such a case, we can argue that the results of CRRC analysis of the dispersed-settled cells are tissue-relevant without any further proof.

We developed the following method for CRRC of intact cultured small multicellular spheroids (Figure 2), which is a modification of the general approach shown in Figure 1. The spheroids are allowed to settle on the surface support, a reaction substrate is added to the media, and the cellular reaction is followed with Z-stack fluorescence confocal imaging covering the entire height of the spheroids. Images of every individual cell are identified in every X-Y plane, integral fluorescence for every cell is determined as a function of time, and kinetic traces of the reaction are built for every cell. Finally, the reaction rate constant is determined from the kinetic traces for every cell, and the kinetic cytometry histogram "number of cells $v s$ rate constant" is built.

For consistency, we used confocal-microscopy-based CRRC not only for spheroids but also for dispersed-settled cells and monolayers. This approach revealed drastic differences between the kinetic histograms of the intact spheroids and the monolayers. 


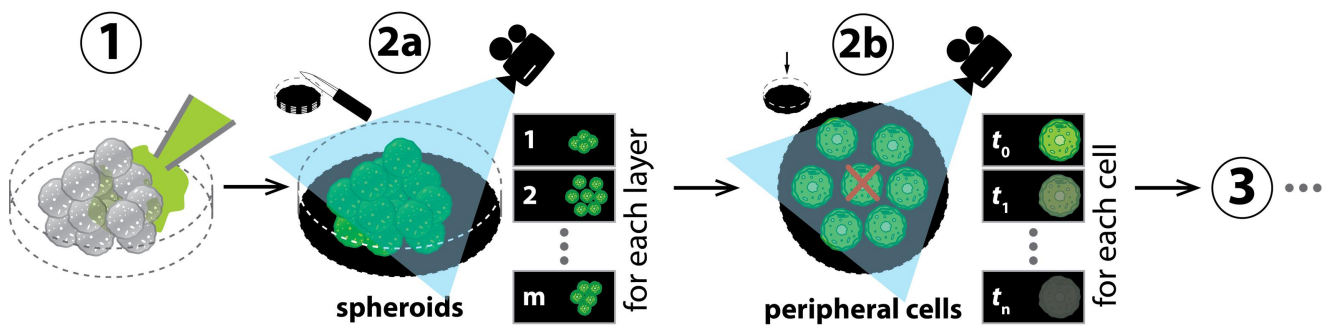

Figure 2. Schematic depiction of CRRC of intact spheroids. The cells are loaded with a fluorogenic (or fluorescent) substrate for the cellular reaction of interest (1). Sequential images of fluorescence intensity from the cells are recorded by time-lapse confocal microscopically in multiple horizontal sections with a $15-\mu \mathrm{m}$ vertical distance between the adjacent horizontal planes (2a). Only cells located in the outer layer of spheroids (outer cells) are analyzed (2b). The subsequent steps (3-5) remain the same as in Figure 1.

The kinetic histograms of the dispersed-settled cells were similar to those of the intact spheroids, and this similarity remained for as long as $24 \mathrm{~h}$ after spheroid disintegration. With regards to MDR transport, these results imply that MDR can be reliably studied by CRRC using dispersed-settled cells obtained by disintegrating a tissue sample. This conclusion is expected to have important implications in the field of chemoresistance prediction. On a more general scale, our results indicate that the spheroid-based approach is practical for assessing tissue relevance of CRRC analysis of the dispersed-settled cells for other types of cells and cellular reactions.

\section{EXPERIMENTAL SECTION}

Monolayer and Spheroid Cell Cultures. Monolayers of human ovarian carcinoma cells $\mathrm{A} 2780^{30}$ were grown under standard cell culture conditions in Dulbecco's Modified Eagle's medium (DMEM, containing $4.5 \mathrm{~g} / \mathrm{L}$ glucose, $1.5 \mathrm{~g} / \mathrm{L}$ sodium bicarbonate, $1 \mathrm{mM}$ sodium pyruvate, and $4 \mathrm{mM}$ L-glutamine) at $37^{\circ} \mathrm{C}$ in a humidified atmosphere of $5 \% \mathrm{CO}_{2}$. DMEM produced by ATCC (ATCC $® 30-2002^{\mathrm{TM}}$ ) was purchased from Cedarlane (Burlington, Ontario, Canada). Cell culture medium contained conventional supplements $(100 \mathrm{IU} / \mathrm{mL}$ penicillin, $100 \mu \mathrm{g} / \mathrm{mL}$ streptomycin, and $10 \%$ fetal bovine serum) purchased from Invitrogen (Burlington, Ontario, Canada).

The culturing of small multicellular spheroids was based on the liquid overlay approach, ${ }^{31}$ adapted for ovarian cells. ${ }^{32}$ Briefly, 96 well plates were treated with $100 \mu \mathrm{L}$ of $10 \%$ agarose per well to create a concave non-adhesive surface. After the solidification of agarose, the wells were filled with $100 \mu \mathrm{L}$ of cell suspension $\left(5 \times 10^{3}\right.$ cells $\left./ \mathrm{mL}\right)$ in DMEM and allowed spheroids to form for 2-3 days before their collection. For imaging, spheroids were placed onto coverslips and allowed to settle and attach to the surface for $5 \mathrm{~h}^{33}$ For studying dispersed-settled cells, the spheroids were disintegrated with trypsin/EDTA treatment into a single-cell suspension, ${ }^{34}$ and the resulting cells were allowed to attach to the plate surface during $5 \mathrm{~h}$ (by default) or $24 \mathrm{~h} .{ }^{35}$

Image Acquisition. Imaging of MDR transport (efflux of a fluorescent substrate from the cells) was performed using an FV300 confocal cell imager (Olympus) in the time-lapse mode. Fluorescein (substrate of MDR transport) and glyburide (also known as glibenclamide, inhibitor of MDR transport) were added to the cell media to final concentrations of 1.5 and $10 \mu \mathrm{M}$, respectively. Reagents were obtained from Sigma-Aldrich (Oakville, Ontario, Canada), Fluka AG (Buchs, Switzerland), and BDH ChemicalsLtd. (Poole, England). Loading cells with fluorescein was stopped by removing the extracellular substrate - the cell support medium was gently replaced (with a single wash) with Krebs-Ringer bicarbonate (KRB) buffer supplemented with $10 \mu \mathrm{M}$ glyburide but not with fluorescein. This media replacement initiated passive substrate leakage through the membrane which was monitored for 4-5 microscopy scans.
Finally, MDR-mediated substrate efflux was initiated by replacing the cell support medium with the KRB buffer free of both glyburide and fluorescein. The fluorescence intensity decrease (caused by the MDR transport of fluorescein from the cells) was further monitored in a series of consecutive microscopy scans.

Image acquisition was performed in a time lapse Z-stack mode using a single optical section for dispersed-settled cells or a cell monolayer or with multiple optical sections for intact spheroids. In the latter case, according to conventional practice, ${ }^{36}$ for small spheroids (diameter $\leq 100 \mu \mathrm{m}$ ) 5-7 sections were taken at $15-\mu \mathrm{m}$ steps (Figure 2 and Figure S1). An argon-ion laser (Melles Griot; $\lambda_{\text {exc }}=488 \mathrm{~nm}$; maximum power of $100 \mathrm{~mW}, 10 \%$ power output) was used for fluorescence excitation. An Omega Optical XF75 filter set was used to decouple excitation light from fluorescence light. Imaging scans were done with a $10 \times$ lens; each full image scan took $1.69 \mathrm{~s}$. The dynamic range of imaging was adjusted to prevent signal saturation, and the majority of images were taken with a 600-700 V photomultiplier (PMT) voltage, $1.0 \times$ gain, and $10 \%$ offset. After completion of MDR-mediated substrate efflux imaging, the identification of individual cells was performed using their treatment with propidium iodide and saponin. ${ }^{37}$

Extraction and Analysis of Kinetic Traces. Kinetic traces from in-plane cells (dispersed-settled cells or cultured-monolayer cells) and peripheral spheroid cells were extracted using ImageJ software with the TimeSeries Analyzer V3 plugin (see Note S1 for more details). Traces were then transferred to OriginPro software for kinetic analysis. Passive substrate leakage (in the presence of glyburide in the cell media) was found to be below $10 \%$ of the total substrate efflux (in the absence of glyburide in the cell media), which is characteristic for A2780 cells and some other cell types, ${ }^{6,38,39}$ and was neglected. Thus, the first order rate constant of MDR transport $\left(k_{\mathrm{MDR}}\right)$ was determined simply from an exponential fit of the main segment of the kinetic trace (Figure $3 \mathrm{f}$, after the arrow mark).

Cell Population Analysis. MDR transport of entire cell populations was characterized by kinetic cytometry histograms "number of cells vs $k_{\mathrm{MDR}}$ ". Histograms were plotted in OriginPro software using the Automatic Binning mode. The histograms were first characterized qualitatively by their modality: unimodal with a single peak or bimodal with two peaks. The histograms were then characterized quantitatively by finding $k_{\mathrm{MDR}}$ values for peak maxima as well as the relative size of a cell subpopulation with a greater value $k_{\mathrm{MDR}}$ and a relative MDR-transport activity of this subpopulation (see section "Relative Size and Relative Activity of the MDR-Active (Drug-Resistant) Cell Subpopulation" in Results and Discussion for more details).

\section{RESULTS AND DISCUSSION}

Methodology for CRRC of Intact Multicellular Spheroids. Our first task was to identify a suitable microscopy mode. CRRC 


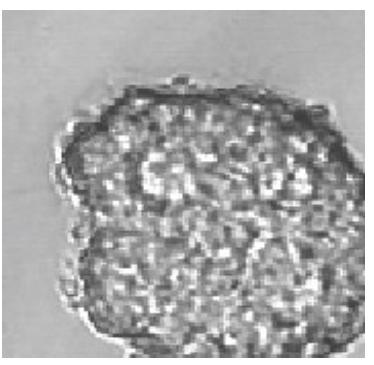

C

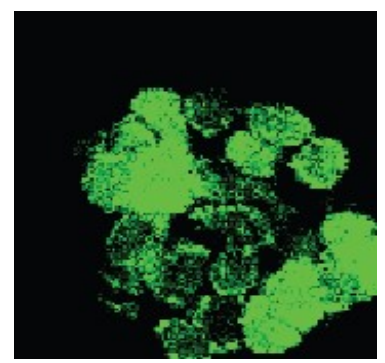

e

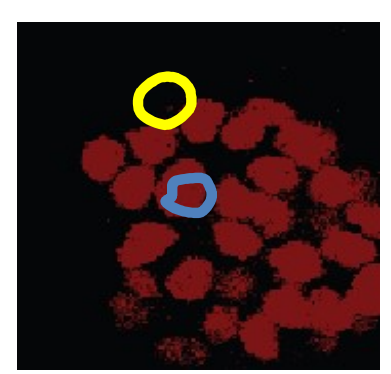

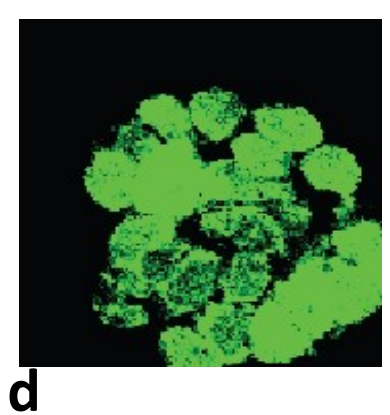
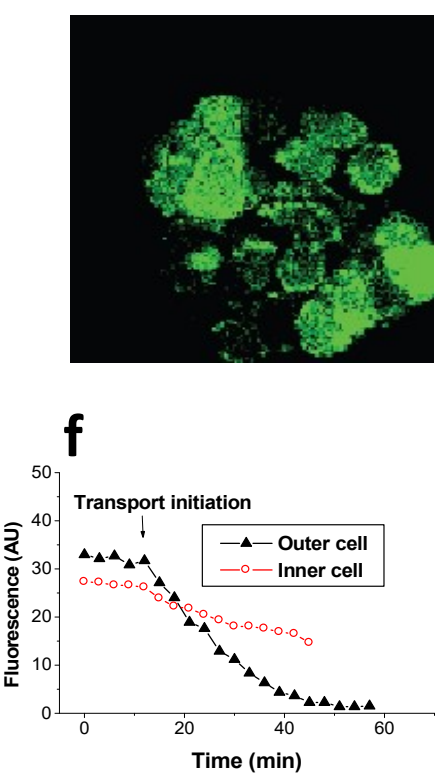

Figure 3. Kinetics of MDR efflux in ovarian-cancer cell spheroids. Representative images of spheroids: a) bright-field; b-d) time-lapse fluorescent scans of the equatorial spheroid plane; e) identification of cells in the equatorial spheroid plane after the completion of acquisition of all kinetic data followed by separation of outer (yellow) and inner (blue) cells; f) typical kinetic traces of fluorescent signal from the outer and inner cells. Start time of MDR-efflux initiation is marked with an arrow; this initiation is achieved through removal of the MDR inhibitor.

of spheroids requires the non-interfering imaging of individual cells in every spheroid. Spheroids are not planar and, therefore, cannot be analyzed at the single-cell level with regular widefield fluorescence microscopy. Therefore, our choice was confocal microscopy, which allows collecting fluorescence information from every cell in every spheroid positioned within the vertical range of scanning. The spheroids must be allowed to settle on the support surface to ensure that all spheroids are imaged within the chosen vertical range of confocal scanning. The settled spheroids also keep their position during the manipulations and long-term imaging, which is necessary in CRRC.

Our second task was to choose a cell model for this work having in mind the main goal of comparing the results for disintegrated spheroids to those of intact spheroids (3D state) and cultured monolayers (2D state). Thus, the ideal choice of a cell model for us was a cell line that could be easily grown either as a monolayer or as spheroids depending on the growth conditions. Such cell lines exist, and, advantageously, one of them, A2780 ovarian cancer cells, is also one of the most studied cellular models for MDR. ${ }^{40,41}$ Thus, we chose A2780 ovarian cancer cells as a cell model for this work.

The choice of the cellular reaction was easy: (i) MDR transport has been well studied in A2780 ovarian cancer cells, (ii) much of recent CRRC advance was done using this reaction, and (iii) CRRC of MDR transport has an immediate practical application in the development of chemoresistance predictors. Fluorescein and glyburide were employed as known MDRtransport substrate and inhibitor, respectively, for A2780 cells. ${ }^{42,43}$ Fluorescein concentration $(1.5 \mu \mathrm{M})$ below the $K_{\mathrm{M}}$ value was used to ensure the first-order regime of the MDR transport process; satisfying this condition allowed us to fit kinetic traces with the exponential-decay equation. ${ }^{44}$

After choosing the microscopy mode, the cell model, and the cellular reaction, we performed CRRC of MDR transport in intact cultured spheroids. Figure 3 demonstrates typical confocal images of spheroids (Figure 3a-e) and representative individual-cell kinetic traces derived from these images (Figure 3f). Activities of MDR transport in individual cells were characterized by values of the first order rate constant $\left(k_{\mathrm{MDR}}\right)$. The values of $k_{\mathrm{MDR}}$ were then assembled into kinetic cytometry histograms showing the distribution of $k_{\mathrm{MDR}}$ over cell populations (discussed in the next section).

An essential feature of spheroids is the suppression of substrate efflux from the inner cells by substrate accumulation in the intercellular space. The substrate accumulates in the intercellular space because it diffuses slowly within the spheroid; diffusivity within a spheroid is only $5-10 \%$ of that in the cell medium. ${ }^{45}$ Compared to substrate efflux from the outer cells, there was sluggishness of substrate efflux from the inner cells: poor response of substrate efflux to MDR-transport initiation (through removal of the MDR inhibitor) is clearly illustrated in Figure 3f. Thus, studying MDR efflux from the entire population of cells within intact spheroids would cause an underestimation of MDR capacity. Such underestimation could lead to misinterpretation of our study results. Thus, in our methodology for CRRC of intact spheroids, the inner cells were excluded from the analysis. Instead, only the outer cells, which were exposed to the bulk medium and showed the full capacity of substrate efflux, were used to define MDR transport in the 3D state.

Influence of Cell-Culture Dimensionally on MDR-Transport Kinetics. Here, we will be comparing the entire cell population of the disintegrated spheroids to the outer-cell subpopulation from the intact spheroids. The following is the justification for this approach. First, a distinctive feature of small spheroids (with a diameter of below $200 \mu \mathrm{m}$ ) is low cell heterogeneity caused by the lack of significant intra-spheroidal gradients of metabolites. ${ }^{46}$

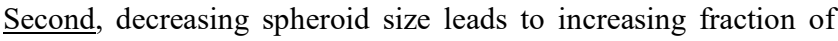
the outer cells in the spheroid cell population. Specifically, the ratio between the number of outer cells and the total number of cells is close to the ratio between the volume of the surface cell layer and the total volume of the spheroid. The latter ratio is equal to $1-(R-r)^{3} / R^{3}$, where $R$ is the spheroid radius and $r$ is the thickness of the outer cell layer. Based on this formula, for a typical spheroid of $100 \mu \mathrm{m}$ in diameter with a $15-\mu \mathrm{m}$ thickness of the outer cell layer, ${ }^{47}$ the outer cells constitute approximately $70 \%$ of the entire cell population in the spheroid. Third, comparing all dispersed spheroidal cells (which include inner and outer cells) to the outer cells in the intact spheroids can reveal the reason for the observed sluggishness of substrate efflux from the inner cells (Figure 3f). If the histogram of all dispersed-settled cells is similar to that of the outer intact-spheroid cells, we can conclude that the slow efflux from the inner cells is associated solely with the slow substrate diffusivity in the intercellular space and not with the difference in MDR status between the outer and inner cells.

Figure 4 shows kinetic cytometry histograms for outer cells in intact spheroids (3D state), cultured cell monolayers (2D state), and dispersed-settled cells obtained by disintegration of spheroids (to be assigned to 3D or 2D states). All experiments shown in this figure were performed at the same time point after plating the cells. The same set of experiments was also repeated at another time point and the results were similar to the one depicted in Figure 4. 


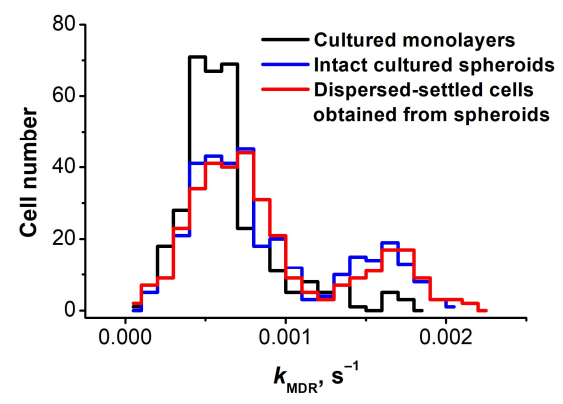

Figure 4. Distribution of $k_{\mathrm{MDR}}$ values for cells within cultured monolayers (black line), the outer cells in cultured spheroids (blue line), and dispersed-settled cells obtained by disintegrating the spheroids and allowing the cells to settle on the surface during a 5-h incubation period (red line). All the cells were from the A2780 ovarian cancer cell line.

The monolayer revealed a unimodal histogram (black line) with a peak maximum at $k_{\mathrm{MDR}} \approx 0.6 \times 10^{-3} \mathrm{~s}^{-1}$. In contrast, the outer cells in intact spheroids (blue line) and the dispersed-settled cells derived from such spheroids (red line) have a bimodal distribution with the first peak being also at $k_{\mathrm{MDR}} \approx 0.6 \times 10^{-3} \mathrm{~s}^{-1}$ and the second peak at $k_{\mathrm{MDR}} \approx 1.6 \times 10^{-3} \mathrm{~s}^{-1}$.

The observed difference between the $2 \mathrm{D}$ state (monolayers) and 3D state (intact spheroids) is qualitative; these histograms can serve as reference standards for determining the dimensionality of the dispersed-settled cells. The difference between the outers cells in intact spheroids and dispersed-settled cells is minor quantitative clearly showing that the dispersed-settled cells maintain MDR transport of the 3D state for at least $5 \mathrm{~h}$ that passed between spheroid disintegration and microscopy measurements. Thus, the dispersed-settled cells can be unambiguously assigned to the $3 \mathrm{D}$ state. Moreover, since the histogram of all dispersed-settled cells is similar to that of the outer intact-spheroid cells, we can conclude that the observed slow efflux from the inner cells (Figure 3f) is solely due to the slow substrate diffusivity in the intercellular space and not due to the difference in MDR status between the outer and inner cells.

The highly pronounced bimodal nature of the spheroidal-cell histograms was somewhat unexpected. It suggests that the spheroidal cells have a significant subpopulation of cells with an elevated level of MDR-transport activity. Importantly, this heterogeneity cannot be ascribed to the difference in cell's location in the spheroids as we considered only the outer cells. This heterogeneity is likely associated with the general increase of cellular heterogeneity in spheroids. For instance, it is known that 3D culturing causes enrichment of subpopulation of tumorinitiating cells. ${ }^{48}$ It is worth mentioning that when comparing our results with the literature data, we found that the range of $k_{\mathrm{MDR}}$ variation observed by us in A2780 cells, $(0.5-2.5) \times 10^{-3} \mathrm{~s}^{-1}$, was close to those reported for other cell types. ${ }^{38,49,50}$

The revealed difference between MDR-transport kinetics in the spheroidal cells and cultured cell monolayers confirms that cell-population dimensionality plays an important role in MDR transport. Overall, spheroidal cells show a greater median $k_{\mathrm{MDR}}$ value than the monolayers indicating that MDR transport may be a major contributor to the increased cumulative drug resistance in the $3 \mathrm{D}$ state. ${ }^{51,52}$ In addition, the revealed difference between the intact spheroids and cell monolayers is qualitative. It provides a perfect means for comparative phenotype assessment of the dispersed-settled cells. This comparison is the basis for a conclusion on the span of MDR-transport maintenance in the dispersed-settled cells and, thus, for assessing tissue relevance of CRRC analysis of such cells.

The demonstrated long-term maintenance of the 3D state by the dispersed cells may help to create hybrid assay systems combining higher physiological relevance of 3D cellular aggregates with the significant practical advantages of 2D-based assays. ${ }^{53}$ From the theoretical standpoint, knowing the span of the
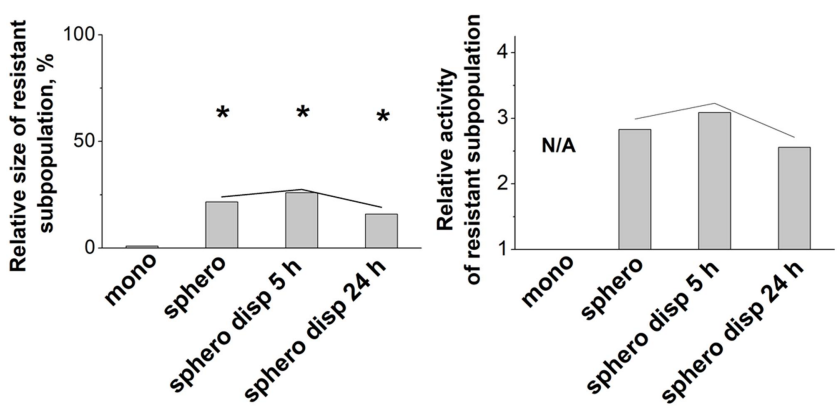

Figure 5. Effect of cell culture dimensionality on the heterogeneity of MDR-transport activity with respect to relative size (RS, left panel) and relative activity (RA, right panel) in A2780 ovarian cancer cells. Abbreviations used are: mono, monolayers; sphero, spheroids; sphero disp $5 \mathrm{~h}$, dispersed-settled cells $5 \mathrm{~h}$ after spheroid disintegration; sphero disp $24 \mathrm{~h}$, dispersed-settled cells $24 \mathrm{~h}$ after spheroid disintegration. The asterisks denote statistical significance (at the $P<0.05$ level) of the difference between parameters of spheroidal and monolayer cells. Statistical significance was not available (N/A) for the RA right panel as the drug-sensitive subpopulaton dominated the cell monolayers.

phenotype maintenance in the dispersed cells may help to better understand the modulation of cellular state in $3 \mathrm{D}$ cell culture. ${ }^{54}$ Our next goal was to conduct detailed statistical analysis of $k_{\mathrm{MDR}}$ distributions upon cells' transition from 3D to 2D states.

Relative Size and Relative Activity of the MDR-Active (Drug-Resistant) Cell Subpopulation. Experimental and modeling studies showed that drug resistance in cell populations correlates with the ratio between drug-resistant and drug-sensitive subpopulations. ${ }^{55}$ Accordingly, our recent work on the application of CRRC to MDR transport focused on accurately finding relative size (RS) and relative activity (RA) of the MDR-active (drugresistant) cell subpopulation. RS was previously defined as a ratio between cell numbers in the drug-resistant and drug-sensitive (main) subpopulations. ${ }^{6}$ Here, we redefine RS as a fraction of the drug-resistant subpopulation, e.g. the ratio between the number of cells in the drug-resistant subpopulation and the total number of cells in the population. The new definition of RS is less prone to errors since the denominator is a constant; further, it allows us to characterize more than two sub-populations of cells in terms of their fractions within the total population. RA was defined as a ratio between the median $k_{\mathrm{MDR}}$ values in the drug-resistant and drug-sensitive subpopulations. ${ }^{6}$ We keep this definition as $k_{\mathrm{MDR}}$ is not an additive parameter, unlike the number of cells. Both drugsensitive and drug-resistant subpopulations were clearly visible in the spheroidal cells, while the drug-sensitive subpopulation completely dominated the cell monolayers (Figure 4).

Figure 5 provides a statistical analysis of 3D and 2D MDR measurements depicted in Figure 4 in terms of RS (left panel) and RA (right panel) using a t-test. The histograms in Figure 4 and the data for RS and RA in Figure 5 clearly demonstrate the preservation of the main 3D-related feature of the MDR-activity distribution - bimodality - in the dispersed-settled spheroidal cells. A slight trend for increase in RS and RA for dispersedsettled cells after $5 \mathrm{~h}$ may reflect the buffering effect of the spheroid interior on the probe efflux from its surface. However, the extension of the post-dispersion period to $24 \mathrm{~h}$ leads to some progression of RS and RA towards those of the 2D-state.

Potential Mechanism and Consequences of the Formation of MDR-Active Subpopulation in the 3D State. Kinetics of MDR transport within spheroids has thus far been examined twice. In one case ${ }^{56}$ the study measured changes of fluorescentsubstrate signal inside large spheroid $(60-80 \mu \mathrm{m}$ under the surface) where the cells have no contact with bulk medium and substrate diffusivity is reduced. Therefore, the observed substrate efflux should have been an underestimation of the full 3D MDR- 
transport capacity. In the second case, $\mathrm{Z}$ confocal stacks were analysed using a maximal intensity projection, i.e. fluorescence intensity of entire spheroid was measured rather than that of individual spheroid cells. ${ }^{57}$ Our current work provides analysis of MDR transport under 3D conditions performed at the single-cell level and with minimal optical and diffusional obstacles, and, thus, it overcomes the limitations of the previous measurements.

Tumor cell spheroids are gaining popularity as a tool for designing and testing chemotherapeutic protocols. ${ }^{58}$ Features specific for 3D-grown cells and distinguishing these cells from the monolayer cells originate from two sources: (i) cell-cell and cellintercellular matrix interactions and (ii) gradients of oxygen and metabolites along the spheroid radius. Our data obtained with small spheroids indicate that cell-cell and cell-intercellular matrix interactions are sufficient to stimulate MDR activity and heterogeneity. Induction of MDR bimodality by $3 \mathrm{D}$ culturing is in line with a well-known increase of heterogeneity in 3D cellular aggregates, specifically, increasing the size of the subpopulation of tumor-initiating cells. ${ }^{59}$

Spheroids formed by the clonal cancer cells and used in this work are the most widely used and studied 3D model of cancer. The subpopulation of cells with the basal level of MDR-transport activity dominates these spheroids, making them relevant to clinical samples in which moderate expression of MDR transporters have been found. ${ }^{60,61}$ On one hand, these spheroids can be considered as a general model of any multicellular malignancy. ${ }^{62}$ On the other hand, they can model well ascitic spheroids in ovarian cancer metastases, pleural spheroids in lung cancer, and circulating spheroids in other cancer types. ${ }^{63-65}$ In particular, ascites containing a mixture of spheroids and individual cancer cells are present in almost all patients with the recurrent disease and represent a major pathway of cancer dissemination. ${ }^{66}$ In this light, the formation of a drug-resistant subpopulation within spheroids is a clinically important event, which can be determined by CRRC but cannot be detected by currently used assays based on whole spheroids. ${ }^{67}$

\section{CONCLUDING REMARKS}

To summarize, we introduced a methodology for studying intact cultured spheroids by CRRC. This allowed us to compare MDR transport in three cellular models: (i) intact cultured spheroids, (ii) dispersed-settled cells obtained by disintegrating the spheroids, and (iii) cultured cell monolayers.

This comparison revealed a number of biologically important findings. When cultured as a monolayer, A2780 ovarian cancer cells show a unimodal distribution of MDR-transport activity at the basal level. When cultured as spheroids, these cells reveal a bimodal distribution of MDR activity due to the formation of an additional cell subpopulation which has a higher level of MDRtransport activity. The small size of the spheroids used in this study allows us to ascribe all differences seen between the monolayers and spheroids to cell-cell interactions and not to the intraspheroidal heterogeneity.

Our further comparison of cultured spheroids and dispersedsettled cells prepared from the spheroids showed that the dispersed-settled cells maintained their 3D state of intact spheroids for as long as $24 \mathrm{~h}$. This finding confirms that CRRC can be applied reliably to dispersed-settled cells for studying MDR transport. This conclusion will likely be valid for other types of cellular reactions; however, it cannot be automatically transferred to them. Instead, a similar study needs to be performed for other reactions and cell types in order to prove tissue relevance of CRRC analysis of dispersed-settled cells. Among such other reactions of interest are metabolic degradation of xenobiotics and repair of DNA damage caused by xenobiotics; both reactions are involved in additional mechanisms of chemoresistance (other than MDR). ${ }^{68}$ Proving the applicability of CRRC to measuring rate constants of these reactions in the dispersed cells would create additional means for developing reliable predictors of clinical chemoresistance.

Moreover, our results suggest that CRRC may be applied to clinical samples after tumor tissue dispersion into a single-cell suspension in order to assess tumor heterogeneity with regards to MDR transport. The evaluation of patient samples by accurately determining the sizes of cell subpopulations with different levels of MDR activity will be a further step in transforming CRRC from an experimental technique into a practical tool for the development of clinically-applicable chemoresistance predictors.

\section{ASSOCIATED CONTENT}

\section{Supporting Information}

The Supporting Information is available free of charge at NNNNNNNNNNNNNNNNN.

Figure S1, sequential fluorescence images of a multicellular spheroid (A2780 ovarian cancer cells) in horizontal planes spaced vertically; Note S1, extraction of kinetic traces from spheroid ZT stacks (PDF)

Additional Supporting Files:

ImageStacks (zip)

KineticTraces (zip)

KineticHistograms (zip)

\section{AUTHOR INFORMATION}

\section{Corresponding Author}

Sergey N. Krylov - Department of Chemistry and Centre for Research on Biomolecular Interactions, York University, Toronto M3J 1P3, Canada; orcid.org/0000-0003-3270-2130; Email: skrylov@yorku.ca

\section{Authors}

Vasilij Koshkin - Department of Chemistry and Centre for Research on Biomolecular Interactions, York University, Toronto M3J 1P3, Canada; orcid.org/0000-0001-6165-5519;

Mariana Bleker de Oliveira - Department of Chemistry and Centre for Research on Biomolecular Interactions, York University, Toronto M3J 1P3, Canada; orcid.org/0000-00015262-725X;

Chun Peng - Department of Biology and Centre for Research on Biomolecular Interactions, York University, Toronto M3J 1P3, Canada; orcid.org/0000-0002-7581-3167;

Laurie E. Ailles - Princess Margaret Cancer Centre and Department of Medical Biophysics, University of Toronto, Toronto M5G 1L7, Canada; orcid.org/0000-0001-72898882;

Geoffrey Liu - Department of Medicine, Medical Oncology, Princess Margaret Cancer Centre, Toronto M5G 2M9, Canada; orcid.org/0000-0002-2603-7296;

Allan Covens - Sunnybrook Odette Cancer Centre, Toronto M4N 3M5, Canada; orcid.org/0000-0001-7958-8502

\section{Author Contributions}

The manuscript was written through contributions of all authors, and all authors have given approval to the final version of the manuscript before the submission.

\section{Notes}

The authors declare no competing financial interest.

\section{ACKNOWLEDGMENTS}

This work was supported by the Natural Sciences and Engineering Research Council of Canada Discovery (Grant 238990). 


\section{REFERENCES}

(1) Dagogo-Jack, I.; Shaw, A. T. Tumour heterogeneity and resistance to cancer therapies. Nat. Rev. Clin. Oncol. 2018, 15, 81-94.

(2) Tellez-Gabriel, M.; Ory, B.; Lamoureux, F.; Heymann, M. F.; Heymann, D. Tumour Heterogeneity: The Key Advantages of Single-Cell Analysis. Int. J. Mol. Sci. 2016, 17, 2142.

(3) Li, X.; Lewis, M. T.; Huang, J.; Gutierrez, C.; Osborne, C. K.; Wu, M. F.; Hilsenbeck, S. G.; Pavlick, A.; Zhang, X.; Chamness, G. C.; Wong, H.; Rosen, J.; Chang, J. C. Intrinsic resistance of tumorigenic breast cancer cells to chemotherapy. J. Natl. Cancer. Inst. 2008, 100, 672-679.

(4) Dean, M.; Fojo, T.; Bates, S. Tumour stem cells and drug resistance. Nat. Rev. Cancer 2005, 5, 275-284.

(5) Mantzaris, N. V. From single-cell genetic architecture to cell population dynamics: quantitatively decomposing the effects of different population heterogeneity sources for a genetic network with positive feedback architecture. Biophys J. 2007, 92, 4271-4288.

(6) Koshkin, V.; Kochmann, S.; Sorupanathan, A.; Peng, C.; Ailles, L. E.; Liu, G.; Krylov, S. N. Cytometry of Reaction Rate Constant: Measuring Reaction Rate Constant in Individual Cells To Facilitate Robust and Accurate Analysis of Cell-Population Heterogeneity. Anal. Chem. 2019, 91, 4186-4194.

(7) Mabert, K.; Cojoc, M.; Peitzsch, C.; Kurth, I.; Souchelnytskyi, S.; Dubrovska, A. Cancer biomarker discovery: current status and future perspectives. Int. J. Radiat. Biol. 2014, 90, 659-677.

(8) Ludwig, J. A.; Weinstein, J. N. Biomarkers in cancer staging, prognosis and treatment selection. Nat. Rev. Cancer 2005, 5, 845-856.

(9) La Thangue, N. B.; Kerr, D. J. Predictive biomarkers: a paradigm shift towards personalized cancer medicine. Nat. Rev. Clin. Oncol. 2011, $8,587-596$

(10) Bedard, P. L.; Hansen, A. R.; Ratain, M. J.; Siu, L. L. Tumour heterogeneity in the clinic. Nature 2013, 501, 355-364.

(11) Bensalah, K.; Montorsi, F.; Shariat, S. F. Challenges of cancer biomarker profiling. Eur. Urol. 2007, 52, 1601-1609.

(12) Djordjevic, B.; Lange, C. S. Cell-cell interactions in spheroids maintained in suspension. Acta Oncologica 2006, 45, 412-420.

(13) Yu, Y. R.; O'Koren, E. G.; Hotten, D. F.; Kan, M. J.; Kopin, D.; Nelson, E. R.; Que, L.; Gunn, M. D. A Protocol for the Comprehensive Flow Cytometric Analysis of Immune Cells in Normal and Inflamed Murine Non-Lymphoid Tissues. PLoS One 2016, 11, e0150606.

(14) Devaraj, V.; Bose, B. Morphological State Transition Dynamics in EGF-Induced Epithelial to Mesenchymal Transition. J. Clin. Med. 2019, 8,911

(15) Pedraza, J. M.; Paulsson, J. Effects of molecular memory and bursting on fluctuations in gene expression. Science 2008, 319, 339-343.

(16) Nepal, S.; Kumar, P. Dynamics of phenotypic switching of bacterial cells with temporal fluctuations in pressure. Phys. Rev. E 2018, 97, 052411

(17) Rees, D. C.; Johnson, E.; Lewinson, O. ABC transporters: the power to change. Nat. Rev. Mol. Cell. Biol. 2009, 10, 218-227.

(18) Robey, R. W.; Pluchino, K. M.; Hall, M. D.; Fojo, A. T.; Bates, S. E.; Gottesman, M. M. Revisiting the role of $\mathrm{ABC}$ transporters in multidrug-resistant cancer. Nat. Rev. Cancer 2018, 18, 452-464.

(19) Shirasaka, Y.; Onishi, Y.; Sakurai, A.; Nakagawa, H.; Ishikawa, T.; Yamashita, S. Evaluation of human P-glycoprotein (MDR1/ABCB1) ATPase activity assay method by comparing with in vitro transport measurements: Michaelis-Menten kinetic analysis to estimate the affinity of P-glycoprotein to drugs. Biol. Pharm. Bull 2006, 29, 2465-2471.

(20) Koshkin, V.; Ailles, L. E.; Liu, G.; Krylov, S. N. Preservation of the 3D Phenotype Upon Dispersal of Cultured Cell Spheroids Into Monolayer Cultures. J. Cell. Biochem. 2017, 118, 154-162.

(21) Koshkin, V.; Ailles, L. E.; Liu, G.; Krylov, S. N. Metabolic Suppression of a Drug-Resistant Subpopulation in Cancer Spheroid Cells. J. Cell. Biochem. 2016, 117, 59-65.

(22) Koshkin, V.; Yang, B. B.; Krylov, S. N. Kinetics of MDR transport in tumor-initiating cells. PLoS One 2013, 8, e79222.

(23) Koshkin, V.; Krylov, S. N. Single-cell-kinetics approach to discover functionally distinct subpopulations within phenotypically uniform populations of cells. Anal. Chem. 2013, 85, 2578-2581.

(24) Koshkin, V.; Krylov, S. N. Correlation between multi-drug resistance-associated membrane transport in clonal cancer cells and the cell cycle phase. PLoS One 2012, 7, e41368.

(25) Koshkin, V.; Krylov, S. N. Single-cell-kinetics approach to compare multidrug resistance-associated membrane transport in subpopulations of cells. Anal. Chem. 2011, 83, 6132-6134.

(26) Costa, E. C.; Moreira, A. F.; de Melo-Diogo, D.; Gaspar, V. M.; Carvalho, M. P.; Correia, I. J. 3D tumor spheroids: an overview on the tools and techniques used for their analysis. Biotechnol. Adv. 2016, 34 , 1427-1441.

(27) Ryu, N. E.; Lee, S. H.; Park, H. Spheroid Culture System Methods and Applications for Mesenchymal Stem Cells. Cells 2019, 8, 1620.

(28) Chitcholtan, K.; Sykes, P. H.; Evans, J. J. The resistance of intracellular mediators to doxorubicin and cisplatin are distinct in 3D and 2D endometrial cancer. J. Transl. Med. 2012, 10, 38.

(29) Luca, A. C.; Mersch, S.; Deenen, R.; Schmidt, S.; Messner, I.; Schäfer, K. L.; Baldus, S. E.; Huckenbeck, W.; Piekorz, R. P.; Knoefel, W. T.; Krieg, A.; Stoecklein, N. H. Impact of the 3D microenvironment on phenotype, gene expression, and EGFR inhibition of colorectal cancer cell lines. PLoS One 2013, 8, e59689.

(30) Xu, G.; Zhong, Y.; Munir, S.; Yang, B. B.; Tsang, B. K.; Peng, C. Nodal Induces Apoptosis and Inhibits Proliferation in Human Epithelial Ovarian Cancer Cells via Activin Receptor-Like Kinase 7. J. Clin. Endocrinol. Metab. 2004, 89, 5523-5534.

(31) Friedrich, J.; Seidel, C.; Ebner, R.; Kunz-Schughart, L. A Spheroid-based drug screen: considerations and practical approach. Nat. Protoc. 2009, 4, 309-324

(32) Frankel, A.; Buckman, R.; Kerbel, R. S. Abrogation of taxolinduced G2-M arrest and apoptosis in human ovarian cancer cells grown as multicellular tumor spheroids. Cancer Res. 1997, 57, 2388-2393.

(33) Acker, H.; Carlsson, J.; Holtermann, G.; Nederman, T.; Nylén, T. Influence of glucose and buffer capacity in the culture medium on growth and $\mathrm{pH}$ in spheroids of human thyroid carcinoma and human glioma origin. Cancer Res. 1987, 47, 3504-3508.

(34) Filippovich, I. V.; Sorokina, N. I.; Robillard, N.; Chatal, J. F. Radiation-induced apoptosis in human ovarian carcinoma cells growing as a monolayer and as multicell spheroids. Int. J. Cancer 1997, 72, 851-859.

(35) Huehls, A. M.; Huntoon, C. J.; Joshi, P. M.; Baehr, C. A.; Wagner, J. M.; Wang, X.; Lee, M. Y.; Karnitz, L. M. Genomically Incorporated 5Fluorouracil that Escapes UNG-Initiated Base Excision Repair Blocks DNA Replication and Activates Homologous Recombination. Mol. Pharmacol. 2016, 89, 53-62.

(36) Wartenberg, M.; Hescheler, J.; Acker, H.; Diedershagen, H.; Sauer, H. Doxorubicin distribution in multicellular prostate cancer spheroids evaluated by confocal laser scanning microscopy and the "optical probe technique". Cytometry 1998, 31, 137-145.

(37) Yeh, C.-J. G.; Hsi, B.-L.; Page Faulk, W. Propidium iodide as a nuclear marker in immunofluorescence. II. Use with cellular identification and viability studies. J. Immunol. Methods 1981, 43, 269-275.

(38) Prosperi, E.; Croce, A. C.; Bottiroli, G.; Supino, R. Flow cytometric analysis of membrane permeability properties influencing intracellular accumulation and efflux of fluorescein. Cytometry 1986, 7, $70-75$.

(39) Saengkhae, C.; Loetchutinat, C.; Garnier-Suillerot, A. Kinetic analysis of fluorescein and dihydrofluorescein effluxes in tumour cells expressing the multidrug resistance protein, MRP1. Biochem. Pharmacol. 2003, 65, 969-977.

(40) Henkels, K. M.; Turchi, J. J. Induction of apoptosis in cisplatinsensitive and -resistant human ovarian cancer cell lines. Cancer Res. 1997, $57,4488-4492$

(41) Hallas-Potts, A.; Dawson, J. C.; Herrington, C. S. Ovarian cancer cell lines derived from non-serous carcinomas migrate and invade more aggressively than those derived from high-grade serous carcinomas. Sci. Rep. 2019, 9, 5515.

(42) Rychlik, B.; Balcerczyk, A.; Klimczak, A.; Bartosz, G. The role of multidrug resistance protein 1 (MRP1) in transport of fluorescent anions across the human erythrocyte membrane. J. Membr. Biol. 2003, 193, 7990.

(43) Payen, L.; Delugin, L.; Courtois, A.; Trinquart, Y.; Guillouzo, A.; Fardel, $\mathrm{O}$. The sulphonylurea glibenclamide inhibits multidrug resistance protein (MRP1) activity in human lung cancer cells. Br. J. Pharmacol. 2001, 132, 778-784.

(44) Perrin, C. L. Linear or Nonlinear Least-Squares Analysis of Kinetic Data? J. Chem. Educ. 2017, 94, 669-672.

(45) Modok, S.; Scott, R.; Alderden, R. A.; Hall, M. D.; Mellor, H. R.; Bohic, S.; Roose, T.; Hambley, T. W.; Callaghan, R. Transport kinetics of four- and six-coordinate platinum compounds in the multicell layer tumour model. Br. J. Cancer 2007, 97, 194-200.

(46) Durand, R. E. Flow cytometry studies of intracellular adriamycin in multicell spheroids in vitro. Cancer Res. 1981, 41, 3495-3498.

(47) Keithley, R. B.; Weaver, E. M.; Rosado, A. M.; Metzinger, M. P.; Hummon, A. B.; Dovichi, N. J. Single cell metabolic profiling of tumor mimics. Anal. Chem. 2013, 85, 8910-8918.

(48) Herheliuk, T.; Perepelytsina, O.; Ugnivenko, A.; Ostapchenko, L.; Sydorenko, M. Investigation of multicellular tumor spheroids enriched for a cancer stem cell phenotype. Stem Cell Investig. 2019, 6, 21. 
(49) Sardini, A.; Mintenig, G. M.; Valverde, M. A.; Sepúlveda, F. V.; Gill, D. R.; Hyde, S. C.; Higgins, C. F.; McNaughton, P. A. Drug efflux mediated by the human multidrug resistance $\mathrm{P}$-glycoprotein is inhibited by cell swelling. J. Cell Sci. 1994, 107, 3281-3290.

(50) McAlroy, H. L.; Bovell, D. L.; Plumb, J. A.; Thompson, P.; Wilson, S. M. Drug extrusion, 125I- efflux and the control of intracellular [Ca2+] in drug-resistant ovarian epithelial cells. Exp. Physiol. 1999, 84, 285-297.

(51) Barrera-Rodríguez, R.; Fuentes, J. M. Multidrug resistance characterization in multicellular tumour spheroids from two human lung cancer cell lines. Cancer Cell Int. 2015, 15, 47.

(52) Torisawa, Y.-s.; Shiku, H.; Yasukawa, T.; Nishizawa, M.; Matsue, T. Multi-channel 3-D cell culture device integrated on a silicon chip for anticancer drug sensitivity test. Biomaterials 2005, 26, 2165-2172.

(53) Nunes, A. S.; Costa, E. C.; Barros, A. S.; de Melo-Diogo, D.; Correia, I. J. Establishment of 2D Cell Cultures Derived From 3D MCF-7 Spheroids Displaying a Doxorubicin Resistant Profile. Biotechnol. J. 2019, 14, 1800268 .

(54) Mansoori, B.; Mohammadi, A.; Davudian, S.; Shirjang, S.; Baradaran, B. The Different Mechanisms of Cancer Drug Resistance: A Brief Review. Adv. Pharm. Bull. 2017, 7, 339-348.

(55) Howard, G. R.; Johnson, K. E.; Rodriguez Ayala, A.; Yankeelov, T. E.; Brock, A. A multi-state model of chemoresistance to characterize phenotypic dynamics in breast cancer. Sci. Rep. 2018, 8, 12058.

(56) Wartenberg, M.; Frey, C.; Diedershagen, H.; Ritgen, J.; Hescheler, J.; Sauer, H. Development of an intrinsic P-glycoprotein-mediated doxorubicin resistance in quiescent cell layers of large, multicellular prostate tumor spheroids. Int. J. Cancer 1998, 75, 855-863.

(57) Letzsch, S.; Böttcher, K.; Kelm, J.; Messner, S. Quantifying Efflux Activity in 3D Liver Spheroids. Genet. Eng. Biotechn. N. 2015, 35, 14-15.

(58) Mehta, G.; Hsiao, A. Y.; Ingram, M.; Luker, G. D.; Takayama, S. Opportunities and challenges for use of tumor spheroids as models to test drug delivery and efficacy. J. Control. Release 2012, 164, 192-204.

(59) Ishiguro, T.; Ohata, H.; Sato, A.; Yamawaki, K.; Enomoto, T.; Okamoto, K. Tumor-derived spheroids: Relevance to cancer stem cells and clinical applications. Cancer Sci. 2017, 108, 283-289.
(60) Allen, J. D.; Brinkhuis, R. F.; van Deemter, L.; Wijnholds, J.; Schinkel, A. H. Extensive contribution of the multidrug transporters Pglycoprotein and Mrp1 to basal drug resistance. Cancer Res. 2000, 60, 5761-5766.

(61) Calcagno, A. M.; Ambudkar, S. V. Molecular mechanisms of drug resistance in single-step and multi-step drug-selected cancer cells. Methods Mol. Biol. 2010, 596, 77-93.

(62) Perche, F.; Torchilin, V. P. Cancer cell spheroids as a model to evaluate chemotherapy protocols. Cancer Biol. Ther. 2012, 13, 12051213.

(63) Burleson, K. M.; Boente, M. P.; Pambuccian, S. E.; Skubitz, A. P. Disaggregation and invasion of ovarian carcinoma ascites spheroids. $J$. Transl. Med. 2006, 4, 6.

(64) Mancini, R.; Giarnieri, E.; De Vitis, C.; Malanga, D.; Roscilli, G.; Noto, A.; Marra, E.; Laudanna, C.; Zoppoli, P.; De Luca, P.; Affuso, A.; Ruco, L.; Di Napoli, A.; Mesiti, G.; Aurisicchio, L.; Ricci, A.; Mariotta, S.; Pisani, L.; Andreetti, C.; Viglietto, G., et al. Spheres derived from lung adenocarcinoma pleural effusions: molecular characterization and tumor engraftment. PLoS One 2011, 6, e21320.

(65) Denes, V.; Lakk, M.; Makarovskiy, A.; Jakso, P.; Szappanos, S.; Graf, L.; Mandel, L.; Karadi, I.; Geck, P. Metastasis blood test by flow cytometry: in vivo cancer spheroids and the role of hypoxia. Int. J. Cancer. 2015, 136, 1528-1536.

(66) Ahmed, N.; Stenvers, K. L. Getting to know ovarian cancer ascites: opportunities for targeted therapy-based translational research. Front. Oncol. 2013, 3, 256.

(67) Jove, M.; Spencer, J. A.; Hubbard, M. E.; Holden, E. C.; O'Dea, R. D.; Brook, B. S.; Phillips, R. M.; Smye, S. W.; Loadman, P. M.; Twelves, C. J. Cellular Uptake and Efflux of Palbociclib In Vitro in Single Cell and Spheroid Models. J. Pharmacol. Exp. Ther. 2019, 370, 242-251.

(68) Hasan, S.; Taha, R.; Omri, H. E. Current Opinions on Chemoresistance: An Overview. Bioinformation 2018, 14, 80-85. 


\title{
A Spheroid-Based Approach to Assess Tissue Relevance of Analysis of Dispersed-Settled Tissue Cells by Cytometry of Reaction Rate Constant
}

\author{
Vasilij Koshkin, Mariana Bleker de Oliveira, Chun Peng, Laurie E. Ailles, Geoffrey Liu, Allan Covens, and
} Sergey N. Krylov*

Table of Contents

Section

Page

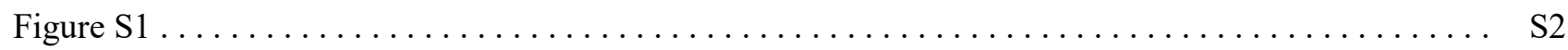

Note S1: Extraction of kinetic traces from spheroid ZT stacks $\ldots \ldots \ldots \ldots \ldots \ldots \ldots \ldots \ldots \ldots \ldots$ S2

\section{Other Supporting Files}

File Name

ImageStacks.zip

KineticTraces.zip

KineticHistograms.zip

\section{Description}

$T$ image stacks of monolayer cells (T stacks 1,2$)$ and dispersed-settled spheroidal cells (T stacks 3,4) as well as ZT image stacks of intact spheroids (ZT stacks 1-6) which were used for extraction of kinetic traces utilized an subsequently building kinetic cytometry histograms. Different T stacks and different ZT stack correspond to experiments performed on different days with new samples.

Each T stack contains 60 images spaced $3 \mathrm{~min}$ in time; recording one image took $1.69 \mathrm{~s}$. ZT stacks contain different numbers of images as spheroids varied in size and required different numbers of horizontal sections with different $Z$ positions for every time point. Accordingly ZT stacks 1-6 contain for every time point 8, 10, 7, 6, 9, and 5 images respectively. The numbers of time points in ZT stacks $1-6$ are 13, 14, 8, 10, 10, and 10, respectively. Totals numbers of images in ZT stacks $1-6$ are 104, 140, 56, 60, 90, and 50 , respectively. Completion of single $\mathrm{Z}$ stack took approximately $10 \mathrm{~s}$ and one $\mathrm{Z}$ stack was done every $3 \mathrm{~min}$. The start of every next cycle of images with varying $Z$ can be identified by taking into account the number of images in a $Z$ stack. For example, for ZT stack 1, a Z stack for every time point contain 8 images, accordingly the first $Z$ stack will start with image 1 , the second $Z$ stack will start with image 9 and so on. Similarly, for ZT stack 2, a Z stack for every time point contain 10 images, accordingly the first $Z$ stack will start with image 1 , the second $Z$ stack will start with image 11 and so on.

Kinetic traces of individual spheroid cells, dispersed-settled spheroidal cells and monolayer cells obtained from time dependence of fluorescence intensity calculated from the image stacks. Column $\mathrm{A}(\mathrm{X})$ shows image number in a sequence of consecutive images separated by $3 \mathrm{~min}$; accordingly, this column can be considered as time if the number is multiplied by $3 \mathrm{~min}$. Each column to the right of column $\mathrm{A}(\mathrm{X})$ contains data on time dependence of relative fluorescence intensity from a single cell.

Kinetic cytometry histograms: $k_{\mathrm{MDR}}$ distributions in populations of outer cells in intact spheroids, dispersed-settled spheroidal cells, and monolayer cells. The number of cells in each histogram is 347 . 
a

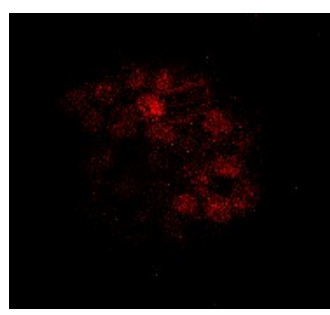

b

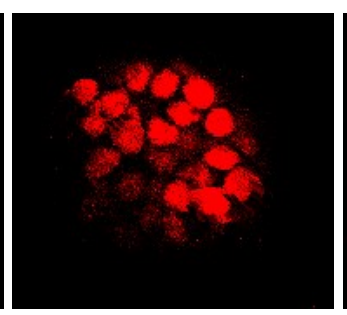

C

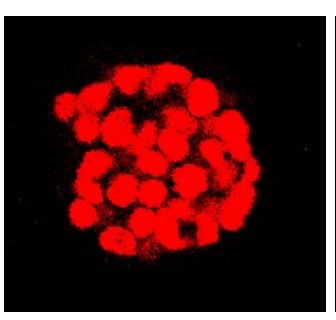

d

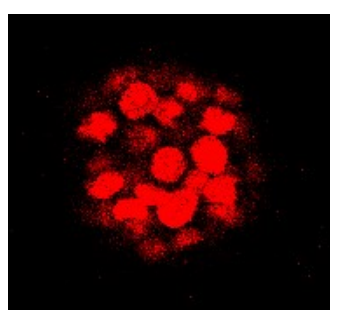

e

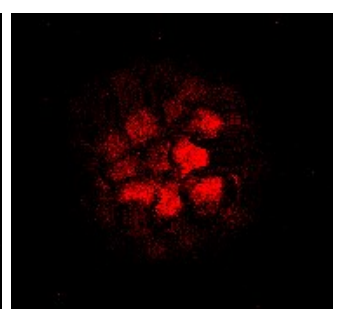

Figure S1. Sequential fluorescence images of a multicellular spheroid (A2780 ovarian cancer cells) in horizontal planes spaced vertically. The distance between the adjacent planes was $15 \mu \mathrm{m}$. Images were taken from the top to the bottom of the spheroid: $a-b)$ images above the equatorial plane; c) image in the equatorial plane; $d-$

e) images below the equatorial plane. Cells' boundaries were identified with propidium iodide staining.

\section{Note S1: Extraction of kinetic traces from spheroid ZT stacks}

Spheroid ZT stacks (archived in ZTseries.zip), were evaluated as specified below:

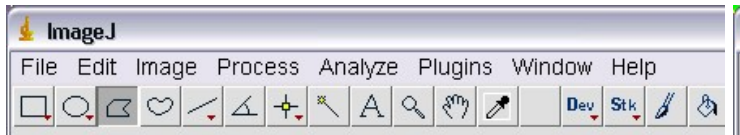

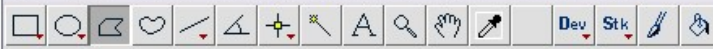
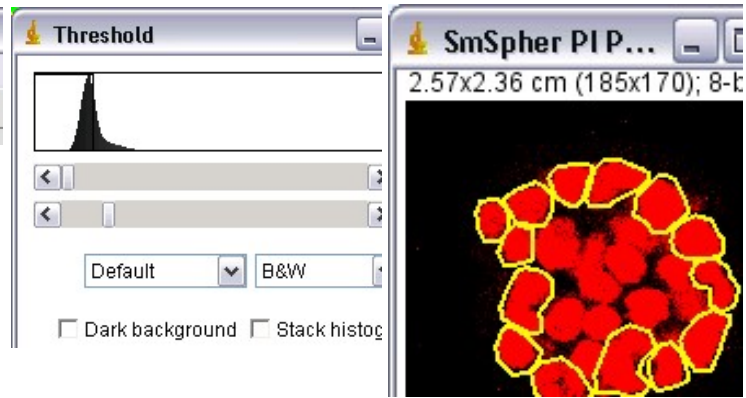

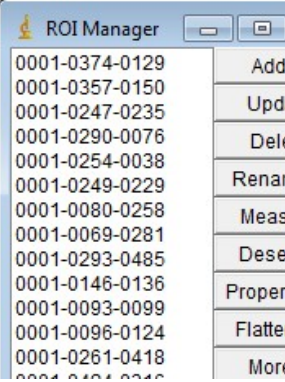

1. To facilitate cell identification, Z stacks of spheroids treated with PI/saponin (after the kinetic MDR assay) were open in ImageJ. Thresholds were adjusted for matching manual cell identification ("gold standard") and applied to individual $\mathrm{Z}$ sections (a typical equatorial section is shown). Oouter cell contours were selected and saved as ROIs (regions of interest) in ROI Manager.

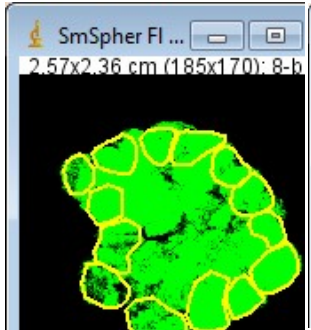

\begin{tabular}{|l|l|l|}
\hline \multicolumn{3}{|l}{ Time Trace(s) } \\
File Edit Font \\
\hline & $0001-0374-0129$ & $0001-0357-0$ \\
\hline 1 & 233.478 & 232.670 \\
2 & 230.851 & 230.284 \\
3 & 225.948 & 220.682 \\
4 & 224.056 & 229.080 \\
5 & 226.746 & 218.352 \\
6 & 219.813 & 221.170 \\
7 & 218.918 & 225.330 \\
8 & 213.675 & 226.784
\end{tabular}

\begin{tabular}{|l|l|l|l|}
\hline \multicolumn{1}{|c|}{} \\
\hline 244.162 & 242.750 & 227.000 & 229.681 \\
244.338 & 238.831 & 216.690 & 228.799 \\
246.764 & 234.355 & 202.886 & 229.208 \\
243.601 & 226.863 & 191.557 & 225.264 \\
235.115 & 228.460 & 190.380 & 221.118 \\
236.230 & 225.194 & 189.835 & 221.243 \\
237.324 & 227.137 & 191.247 & 220.201 \\
233.730 & 224.266 & 190.114 & 216.403
\end{tabular}

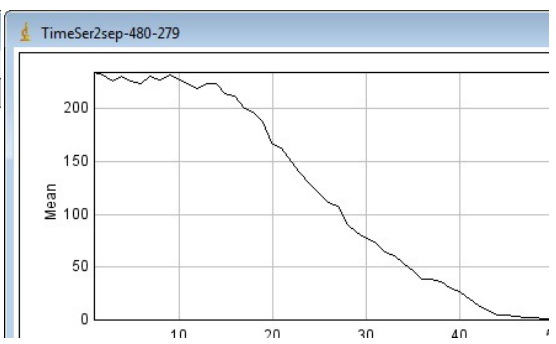

2. ROIs determined in Step 1 were applied to ZT stacks acquired in the process of kinetic MDR assay. Time Series Analyser plugin was used to form time lapse (T) sequence of images for each $Z$ section. Analysis of these sequences produced individual cell (ROI) kinetics in a tabular form and averaged cell population kinetics in a graphical form. 